\title{
MODELLING AND PRODUCTION CONFIGURATION OPTIMIZATION FOR AN ASSEMBLY SHOP
}

\author{
Yang, S. L. ${ }^{* * *, * * *} ;$ Xu, Z. G. ${ }^{* * *} \&$ Wang, J. Y., ${ }^{* * *}$ \\ * Shenyang Institute of Automation, Chinese Academy of Sciences, Shenyang 110016, China \\ ** Institutes for Robotics and Intelligent Manufacturing, Chinese Academy of Sciences, Shenyang \\ 110016, China \\ ${ }^{* * * *}$ University of Chinese Academy of Sciences, Beijing 100049, China \\ E-Mail: yangshengluo@sia.cn
}

\begin{abstract}
Flexible production is able to respond to a changing production system. To efficiently optimize a changed production layout and configuration, a modelling and optimization method based on discrete event simulation (DES) was proposed. A mathematical optimization model of the workshop layout was formulated and solved using a genetic algorithm (GA). In addition, a general modelling method for workshop logistics processes using DES was proposed to efficiently develop a simulation model of the complex production processes. Moreover, the simulation model of the entire production process was constructed using the Plant Simulation software package, and more production factors were considered and optimized. After the optimization process, the logistics volume decreased by $63.5 \%$, and the throughput increased by $42.0 \%$. Additionally, the production process was optimized, and the optimal equipment allocation, worker allocation, buffer allocation, and logistics vehicle allocation were determined. Our work can provide decision guidance and simulation validation for workshop planning.

(Received, processed and accepted by the Chinese Representative Office.)
\end{abstract}

Key Words: Production Performance, Logistics Simulation Modelling, Production Configuration, Plant Simulation, Layout Optimization, Production Process Optimization

\section{INTRODUCTION}

Facing the new industrial revolution, many countries are actively engaged in intelligent manufacturing to establish competitive advantages for their manufacturing industry $[1,2]$. An important characteristic of intelligent manufacturing is flexible production whose corresponding workshop layout and production resource allocation are changeable. Changes in these workshops require a large amount of modelling and analysis to improve the production performance and determine the main production configuration [3].

In a broad sense, production configuration is a process of selecting and configuring appropriate production processes from the identified process elements. This study focuses on determining the main manufacturing resource allocations, such as the equipment allocation, worker allocation, buffer allocation, and logistics vehicle allocation. The determination of these configurations has usually been associated with the optimization of production performance.

Various methods have been used to optimize the production performance, and many algorithms, such as genetic algorithms (GAs), artificial bee colony algorithms, and simulated annealing, have been developed to optimize workshop performance [4-7]. However, Zhang et al. [3] pointed out that it was difficult for these algorithms to address more realistic dynamic problems with uncertain parameters. Moreover, these algorithms suffered from low convergence speeds and inefficiency in complex cases or cases where there were many scenarios to consider.

In addition to these optimization algorithms, value stream mapping (VSM) has been used to eliminate non-value adding activities and increase the service level of manufacturing 
enterprises [8]. Production coordination and the concept of nimble organizations have been used to cope with changes in production [9, 10]. In addition, ARENA software and the Taguchi experimental design method have been applied to optimize the performance of a material handling transfer system [11]. Moreover, visualization support was proposed to improve the design of a manufacturing system [12]. However, more production factors need to be considered to improve the overall production process.

Discrete event simulation (DES) was also used to optimize production performance. DES is a discrete-state and event-driven method for systems whose behaviour is characterized by abrupt changes in the value of its state [13]. DES can be used to efficiently evaluate different production configuration alternatives and production strategies to support decision making [14]. Therefore, DES has been extensively studied to optimize production systems [15-18]. Mainstream DES software includes Plant Simulation, Flexsim, Quest, etc. The Plant Simulation software package, formerly known as eM-plant, has been widely studied because of its powerful logistics simulation functionality.

The simulation and optimization literature on optimizing production performance with the Plant Simulation software package is mainly focused on two aspects: verification and optimization. Verification refers to analysing the performance of proposed algorithms, strategies or improved scenarios; and optimization is the development and implementation of measures to optimize the production layout, logistics strategy, bottleneck process, assembly line balancing, production scheduling, buffer size, equipment configuration, worker configuration, etc.

Qin et al. [19] verified the performance of proposed composite dispatching rules. Yang et al. [20] verified the effectiveness of a modified discrete particle swarm optimization algorithm for solving the multi-object sequencing problem. Grabowik et al. [21] optimized a car suspension manufacturing system by proposing and comparing four improvement scenarios based on an initial simulation. Changes in robots, workers and roller conveyors were simulated to perform a comparative analysis of a production system. Supsomboon and Vajasuvimon [22] compared three alternative strategies for optimizing a job shop production system. Group technology, plant layout, job enlargement and capacity expansion were considered to increase throughput and average worker utilization. Andrade-Gutierrez et al. [23] optimized a flexible die-casting engine-head plant to reduce the production cycle time and increase productivity by identifying the bottlenecks and improving the production process. Huang et al. [24] optimized the facility layout in a flexible manufacturing workshop with the Plant Simulation Layout Optimizer optimization module. Hodoň et al. [25] optimized a production scheduling process by using Plant Simulation to detect wasted resources and assess generated schedules. Supsomboon and Vajasuvimon [26] optimized a production planning process to produce more products with a minimum number of workers.

Based on the above literature, some conclusions are obtained. Few researchers optimized the overall performance of a factory through simulation. Most of them only simulated and optimized certain production factors, such as bottleneck processes or equipment configurations. In addition, few studies have comprehensively considered a facility layout and production process optimization. Moreover, a general simulation modelling method is lacking when using Plant Simulation, especially for logistics modelling, which is one of the most complex and important parts of production system modelling.

This paper seeks to efficiently establish a workshop simulation model to comprehensively optimize production performance and determine the main production configuration. A GA was used to optimize the layout of an assembly shop and Plant Simulation was used to establish and optimize the entire production process of the shop. Moreover, a general modelling methodology for workshop logistics processes was proposed to uniformly describe the logistics processes of all the workstations. In this study, more practical production factors 
were considered and optimized. In addition, the proposed modelling methodology for the logistics process can be used to effectively construct a simulation model of complex production processes. After the optimization process, the logistics volume decreased by $63.5 \%$, the throughput increased by $42.0 \%$, and the optimal production configuration in terms of the equipment allocation, worker allocation, buffer allocation and logistics vehicle allocation, were obtained. This study can provide more efficient, accurate and comprehensive guidance and verification for workshop planning.

\section{METHODS}

\subsection{Mathematical model for layout optimization}

To operate production systems efficiently, factories should not only be operated with optimal planning and operational policies but also have a well-designed facility layout [27]. The facility layout problem (FLP) in this study can be simplified as a quadratic assignment problem (QAP). The QAP can be described as a one-to-one assignment problem of $n$ facilities to $n$ locations. A mathematical model was established with the objective of minimizing the total logistics volume.

The following assumptions are made for the QAP.

(1) The locations for the workstation layout and the distance between these locations are fixed;

(2) The number of workstations equals the number of locations;

(3) Each workstation has the same shape and area.

The objective function is formulated as Eq.(1) [28]:

Subject to:

$$
\min \sum_{i=1}^{n} \sum_{j=1}^{n} \sum_{k=1}^{n} \sum_{l=1}^{n} f_{i k} d_{j l} x_{i j} x_{k l}
$$

$$
\begin{gathered}
\sum_{j=1}^{n} x_{i j}=1, \quad i \in 1,2,3, \ldots, N \\
\sum_{i=1}^{n} x_{i j}=1, \quad j \in 1,2,3, \ldots, N \\
x_{i j}= \begin{cases}1 & , \text { if workstation } i \text { is assigned to location } j \\
0 & , \text { otherwise }\end{cases}
\end{gathered}
$$

where $f_{i k}$ denotes the amount of flow between workstations $i$ and $k$, and $d_{j l}$ denotes the distance between locations $j$ and $l$.

\subsection{Design of a general modelling method for workshop logistics}

A simulation model of the workshop consists mainly of the following modules: workstations, logistics and workers. Logistics is one of the most important and complex modules in modelling.

Based on the common characteristics of the logistics processes in production workshops, a general modelling methodology for workshop logistics processes using Plant Simulation was proposed. The production logistics processes were expressed using transport requests, vehicle movements, loading, unloading and workstation operations. Additionally, a vehicle allocation strategy and optimum path search strategy were designed to assign vehicles to distribution tasks. The distribution tasks were allocated according to task priority and the number of available vehicles. The path optimization was carried out according to the shortest path principle. Finally, the proposed logistics control strategy was implemented using SimTalk, a programming language in Plant Simulation. 
In this study, a vehicle only received one distribution task at a time. In addition, the recording and assignment operations for the transport tasks were carried out using a specified distribution table.

The flow chart of the proposed modelling method for the logistics processes is shown in Fig. 1 A.
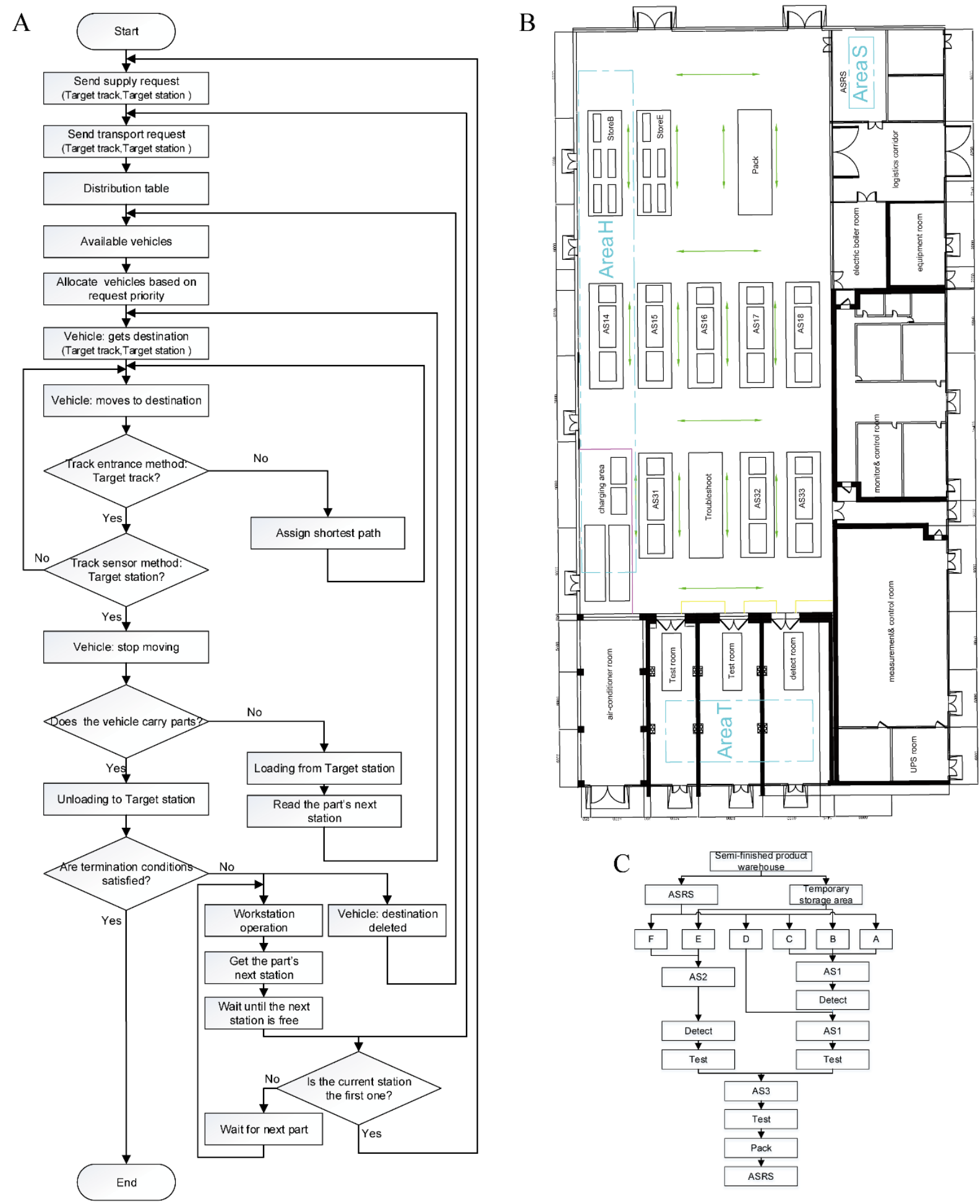

Figure 1: The proposed modelling method and the workshop under study: A) General modelling method of the workshop logistics process for DES, B) Initial layout scheme, C) Process flow chart.

The production logistics process was summarized as the following four processes. 
(1) Transport requests

The transport requests were generated when an assembly operation was completed. For the workstations that operated the first process, a transport request for supplying would also be generated. These requests would be recorded in the distribution table. Then, according to the priority of the distribution task and transport vehicle availability, vehicles were assigned, received the destination, and moved to the destination.

(2) Vehicle movements

The vehicles moved to their destination according to the shortest path principle. Each track contained an entry control method at its entrance and a sensor in the middle. The entry control method determined whether the current track was the target track, and the sensor determined whether the target workstation was within the coverage area of this track. If both of the judgements were true, the current vehicle would stop and carry out the loading or unloading operation. Otherwise, the vehicle would continue to move to their destination.

(3) Loading and unloading

The loading and unloading was performed based on whether the vehicle carried parts. If the vehicle was empty, a loading operation would be performed. Parts would be moved from the target workstation to the vehicle. In addition, the next process for the part would be read and assigned as the current vehicle's next destination. Otherwise, an unloading operation would be performed. The parts would be moved to the target workstation, and the vehicle destination would be deleted.

(4) Workstation operation

After the parts were unloaded, if the termination conditions were not met, the production process would continue. One of the workstation operations, assembly, test or detect would be performed. After the operation, the next procedure for the part would be read, and the next transport request would be sent. Moreover, for the workstations that operated the first process, a transport request for supplying would also be generated.

\section{LAYOUT OPTIMIZATION}

\subsection{The case study: a manual assembly shop}

A manual assembly shop was used as an optimization case study. This shop assembles six types of semi-manufactured parts (A-F) into a final product, through three assembly stages, two inspections and one packaging process. The initial workshop layout and process flow are shown in Fig. $1 \mathrm{~B}$ and Fig. $1 \mathrm{C}$. The semi-finished parts are transported from the semi-finished product warehouse to the assembly shop by a truck. Then, parts B and E are hoisted and transported to the temporary storage area, and the other four parts are hoisted and transported to the Automatic Storage and Retrieval System (ASRS). Five of each part are stored. When the number of parts stored is less than two, a transport request will be sent, and four parts will be transported from the semi-finished product warehouse each time. Inside the workshop, two types of automatic guided vehicles (AGVs) are used to transport the parts to the workstations. The smaller AGV is used for parts A, B, C and D, and the larger AGV is used for part E and other assembled parts.

There are six types of workstations in the assembly shop. Their operation time and the number of workers required are shown in Table I.

\subsection{Modelling and optimization of the layout}

To obtain the optimal layout, a simulation model for the layout optimization was developed using the Plant Simulation software and was based on the established mathematical model. The GA module in Plant Simulation was used to optimize the total logistics volume. 
The workstations in the shop were represented as M01-M17, as shown in Table II. In addition, the distance matrix is shown in Table III, and the annual throughput was assumed to be 1000 units.

Table I: The process time and number of workers required for each workstation.

\begin{tabular}{|c|c|c|c|}
\hline Station & $\begin{array}{c}\text { Number of } \\
\text { stations }\end{array}$ & $\begin{array}{c}\text { Time } \\
(\mathrm{min})\end{array}$ & Workers \\
\hline AS1 & 5 & 156 & 1 \\
\hline AS2 & 4 & 150 & 1 \\
\hline AS3 & 3 & 120 & 1 \\
\hline Detect & 1 & 15 & 1 \\
\hline Test & 2 & 15 & 0.5 \\
\hline Pack & 1 & 45 & 2 \\
\hline
\end{tabular}

Note. 'Workers' is the number of workers required for each workstation. For the test workstations, two workstations were operated by one worker. Hoisting time: $15 \mathrm{~min}$; AGV speed: $0.17 \mathrm{~m} / \mathrm{s}$; Truck speed: $5.56 \mathrm{~m} / \mathrm{s}$.
Table II: Workstation representation.

\begin{tabular}{|c|c||c|c|}
\hline Code & Station & Code & Station \\
\hline M01 & AS14 & M10 & Test1 \\
\hline M02 & AS15 & M11 & Test2 \\
\hline M03 & AS16 & M12 & Pack \\
\hline M04 & AS17 & M13 & Charge \\
\hline M05 & AS18 & M14 & StoreB \\
\hline M06 & AS31 & M15 & StoreE \\
\hline M07 & AS32 & M16 & ASRS \\
\hline M08 & AS33 & M17 & TroShoot \\
\hline M09 & Detect & & \\
\hline
\end{tabular}

Table III: The distance matrix.

\begin{tabular}{|c|c|c|c|c|c|c|c|c|c|c|c|c|c|c|c|c|c|}
\hline Location & $\mathbf{1}$ & $\mathbf{2}$ & $\mathbf{3}$ & $\mathbf{4}$ & $\mathbf{5}$ & $\mathbf{6}$ & $\mathbf{7}$ & $\mathbf{8}$ & $\mathbf{9}$ & $\mathbf{1 0}$ & $\mathbf{1 1}$ & $\mathbf{1 2}$ & $\mathbf{1 3}$ & $\mathbf{1 4}$ & $\mathbf{1 5}$ & $\mathbf{1 6}$ & $\mathbf{1 7}$ \\
\hline $\mathbf{1}$ & 0 & 4 & 8 & 9 & 5 & 9 & 13 & 17 & 14 & 10 & 14 & 18 & 22 & 19 & 15 & 23 & 29 \\
\hline $\mathbf{2}$ & 4 & 0 & 4 & 13 & 9 & 5 & 9 & 13 & 18 & 14 & 10 & 14 & 18 & 23 & 19 & 19 & 25 \\
\hline $\mathbf{3}$ & 8 & 4 & 0 & 17 & 13 & 9 & 5 & 9 & 22 & 18 & 14 & 10 & 14 & 27 & 23 & 15 & 21 \\
\hline $\mathbf{4}$ & 9 & 13 & 17 & 0 & 4 & 8 & 12 & 16 & 5 & 9 & 13 & 17 & 21 & 10 & 14 & 22 & 28 \\
\hline $\mathbf{5}$ & 5 & 9 & 13 & 4 & 0 & 4 & 8 & 12 & 9 & 5 & 9 & 13 & 17 & 14 & 10 & 18 & 24 \\
\hline $\mathbf{6}$ & 9 & 5 & 9 & 8 & 4 & 0 & 4 & 8 & 13 & 9 & 5 & 9 & 13 & 18 & 14 & 14 & 20 \\
\hline $\mathbf{7}$ & 13 & 9 & 5 & 12 & 8 & 4 & 0 & 4 & 17 & 13 & 9 & 5 & 9 & 22 & 18 & 10 & 16 \\
\hline $\mathbf{8}$ & 17 & 13 & 9 & 16 & 12 & 8 & 4 & 0 & 21 & 17 & 13 & 9 & 5 & 26 & 22 & 14 & 12 \\
\hline $\mathbf{9}$ & 14 & 18 & 22 & 5 & 9 & 13 & 17 & 21 & 0 & 4 & 8 & 12 & 16 & 5 & 9 & 17 & 23 \\
\hline $\mathbf{1 0}$ & 10 & 14 & 18 & 9 & 5 & 9 & 13 & 17 & 4 & 0 & 4 & 8 & 12 & 9 & 5 & 13 & 19 \\
\hline $\mathbf{1 1}$ & 14 & 10 & 14 & 13 & 9 & 5 & 9 & 13 & 8 & 4 & 0 & 4 & 8 & 13 & 9 & 9 & 15 \\
\hline $\mathbf{1 2}$ & 18 & 14 & 10 & 17 & 13 & 9 & 5 & 9 & 12 & 8 & 4 & 0 & 4 & 17 & 13 & 5 & 11 \\
\hline $\mathbf{1 3}$ & 22 & 18 & 14 & 21 & 17 & 13 & 9 & 5 & 16 & 12 & 8 & 4 & 0 & 21 & 17 & 9 & 7 \\
\hline $\mathbf{1 4}$ & 19 & 23 & 27 & 10 & 14 & 18 & 22 & 26 & 5 & 9 & 13 & 17 & 21 & 0 & 4 & 12 & 18 \\
\hline $\mathbf{1 5}$ & 19 & 23 & 27 & 14 & 10 & 14 & 18 & 22 & 9 & 5 & 9 & 13 & 17 & 4 & 0 & 8 & 14 \\
\hline $\mathbf{1 6}$ & 23 & 19 & 15 & 22 & 18 & 14 & 10 & 14 & 17 & 13 & 9 & 5 & 9 & 12 & 8 & 0 & 6 \\
\hline $\mathbf{1 7}$ & 29 & 25 & 21 & 28 & 24 & 20 & 16 & 12 & 23 & 19 & 15 & 11 & 7 & 18 & 14 & 6 & 0 \\
\hline
\end{tabular}

Note. The values are the sum of the distance in the lengthwise and widthwise directions. The width of a location is assumed to be 1 . According to the dimensional scale, the length is 3, the distance between the lengthwise and widthwise directions are 2 and 1 , respectively, with a few exceptions.

Moreover, some constraints are considered: ASRS, test and charge workstations are limited to areas $\mathrm{S}, \mathrm{T}$ and $\mathrm{H}$, respectively, as shown in Fig. $1 \mathrm{~B}$.

Finally, the layout optimization model is shown in Fig. 2 A.

A GA was used to solve the mathematical and simulation models. Fig. 2 B shows that the evolutionary process of the offspring converges after 23 generations. After the optimization process, the total logistics volume was reduced from 442518 to 270626 , which was $63.5 \%$ less than that before the optimization process. The optimal layout is shown in Fig. $2 \mathrm{C}$. 


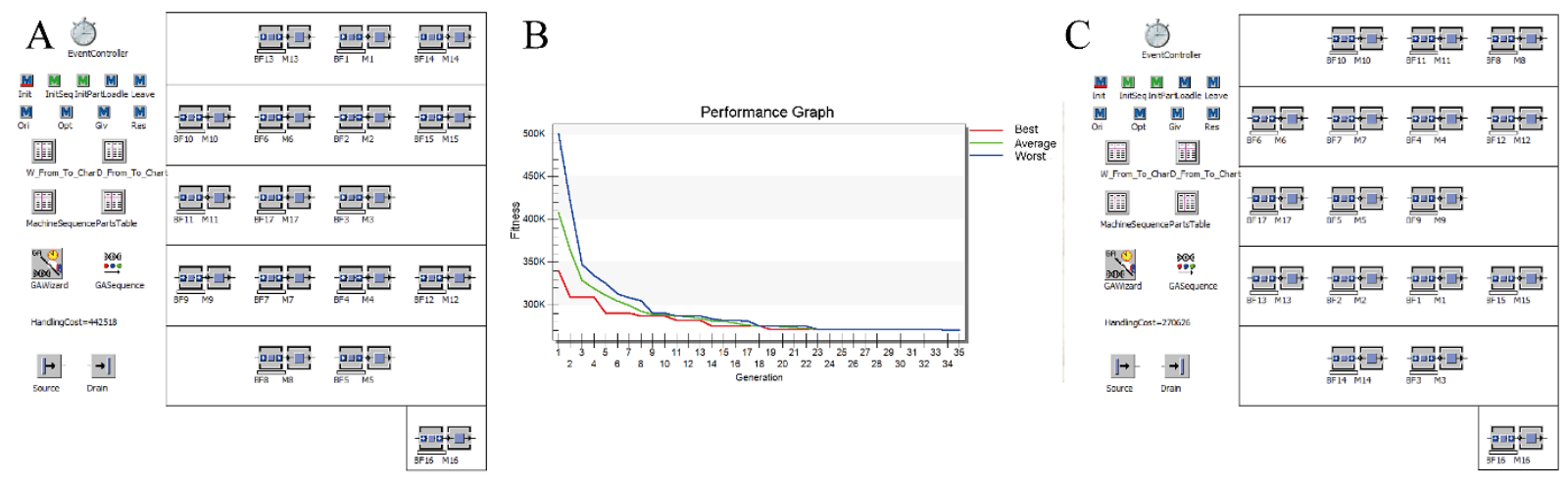

Figure 2: Modelling and optimization of the workshop layout: A) The simulation model for layout optimization, B) Evolution curve of the GA; population $=50$, max. generation $=35$, crossover rate $=0.8$, mutation rate $=0.1, \mathrm{C}$ ) Optimal layout scheme.

\section{MODELLING AND OPTIMIZATION OF THE PRODUCTION PROCESS}

\subsection{Modelling and analysis of the entire production process}

The production simulation model of the entire production process was established using the Plant Simulation software based on the above optimal layout scheme. As shown in Fig. 3, the model mainly includes the following modules: warehousing, workstations, workers, tracks, scheduling strategy, experiment module and statistical analysis.

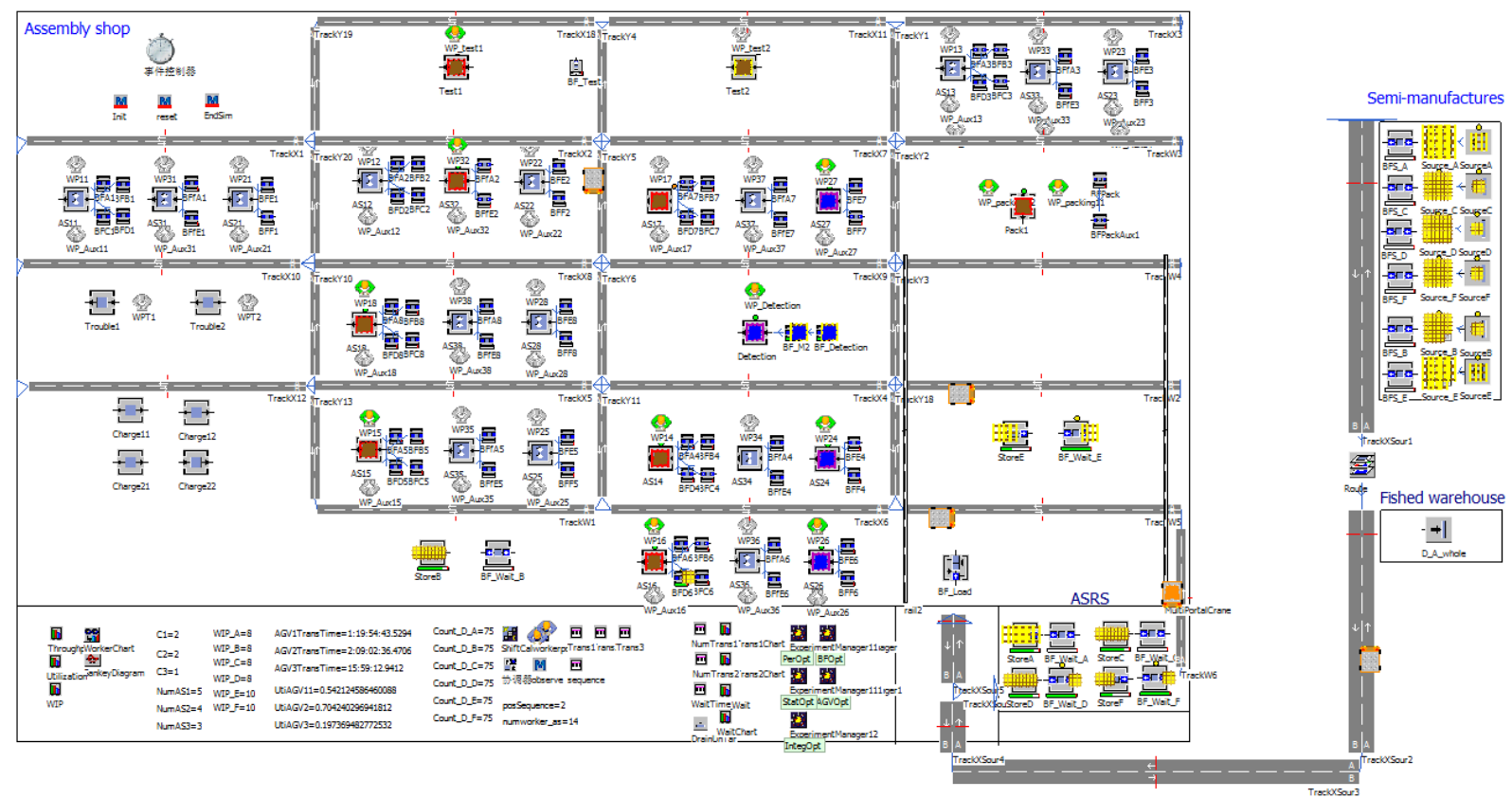

Note. The assembly workstations were designed to be flexible to adapt to future product adjustments. Therefore, each assembly workstation could perform operations AS1-AS3. In this study, three assembly workstations were used for operation AS3, and the others were used for operations AS1 and AS2.

Figure 3: Production simulation model of a manual assembly shop.

The production process was operated 7 hours a day for 22 days a month. After the simulation, the monthly output was 150 units. The facility utilization rates and the worker efficiencies are shown in Fig. $4 \mathrm{~A}$ and Fig. 4 B, respectively.

Fig. $4 \mathrm{~A}$ shows that blockages occurred in many workstations and that the workstation utilization was generally low. Fig. $4 \mathrm{~B}$ shows that 14 main assembly workers were sufficient 
under the current production conditions. Further analysis showed that the blockages occurred mainly because the packaging operational cycle was too long and there were not enough buffers at every workstation. Therefore, the production process and configuration needed to be optimized.

\subsection{Optimization of the production processes and configuration}

According to the above analysis, the workshop production process was optimized through buffer usage and bottleneck elimination. In addition, the optimal allocation of the main production resources was determined through the design of experiment (DOE) method.

In this study, both the number of workers and AGVs were determined to be adequate before they were optimized so that they would not become a limiting factor for an increase in the production volume or further performance improvement.

(1) Buffer size optimization

Among the six types of workstations, buffers were not set at types AS1 and AS2 because there was no extra space at these two types of workstations and they would be replenished as soon as they were idle. In addition, buffers should be set up after the packaging workstation. Therefore, five types of workstations needed buffers.

The DOE method was used to optimize the buffer size where the maximum throughput was used as an evaluation index. A two-level experimental design was employed to improve the computational efficiency. The upper bound of the buffer size was determined in the first stage, and the specified buffer size was determined in the second stage.

In the first stage experiment, the buffer size of each workstation increased from 1 to 5 with an increment of 2 . The results are shown in Fig. $4 \mathrm{~F}$.

The experimental data showed that the minimum upper bound of the buffer size for maximum throughput was $1,1,3,3$ and 1 . Therefore, the second stage experiment, which increased the buffer size from 0 to the corresponding minimum upper bound, was designed. An additional 128 experiments were carried out.

After the buffer optimization, the optimal buffer configuration was 1, 1, 2, 2 and 1. Under this configuration, the monthly output reached 195, which was $30 \%$ higher than that before buffer optimization. In addition, as shown in Fig. $4 \mathrm{C}$, the blockage of each workstation was obviously reduced, and the utilization was greatly improved. Moreover, Fig. $4 \mathrm{G}$ shows that most buffers have relatively high occupation rates and low full occupation rates, indicating that the optimized buffer configuration was reasonable.

(2) Bottleneck process optimization

Fig. $4 \mathrm{C}$ shows that the packaging operation was a bottleneck process, which resulted in much blockage in the original configuration. Because of the low cost of packaging equipment and the extra space in the packaging area, a new packaging workstation was added to eliminate the bottleneck effect.

After the bottleneck process optimization, the monthly output increased from 195 to 213. Moreover, the blockages at the test and detection workstations were significantly reduced, as shown in Fig. 4 D.

However, the utilization of the test workstation was still relatively low. Because of the high price of the test equipment, one test workstation was eliminated. After this reduction, the output did not decrease, although Fig. 4 E shows a slight increase in the blockages at the test and detection workstations. Furthermore, a DOE was carried out, which verified that after the adjustment of the workstations, the aforementioned buffer configuration was still optimal.

(3) Worker quantity optimization

The number of assembly workers was designed to increase from 9 to 18 , while both the number of detect workers and test workers remained at 1 . Therefore, 10 experiments were carried out. Fig. $4 \mathrm{H}$ shows that as the number of assembly workers increased, the output 
increased and reached the maximum when the number of assembly workers was 14 . In addition, the labour intensity of the assembly workers decreased, while that of the test and detect workers increased slightly. Considering the throughput and labour intensity, the optimal number of assembly workers was determined to be 14 .

(4) Transport vehicle quantity optimization

The number of AGV1 and AGV2 vehicles increased from 1 to 5, and the number of trucks increased from 1 to 3 . Therefore, 75 experiments were carried out, and the throughput and vehicle utilization are shown in Fig. $4 \mathrm{I}$.
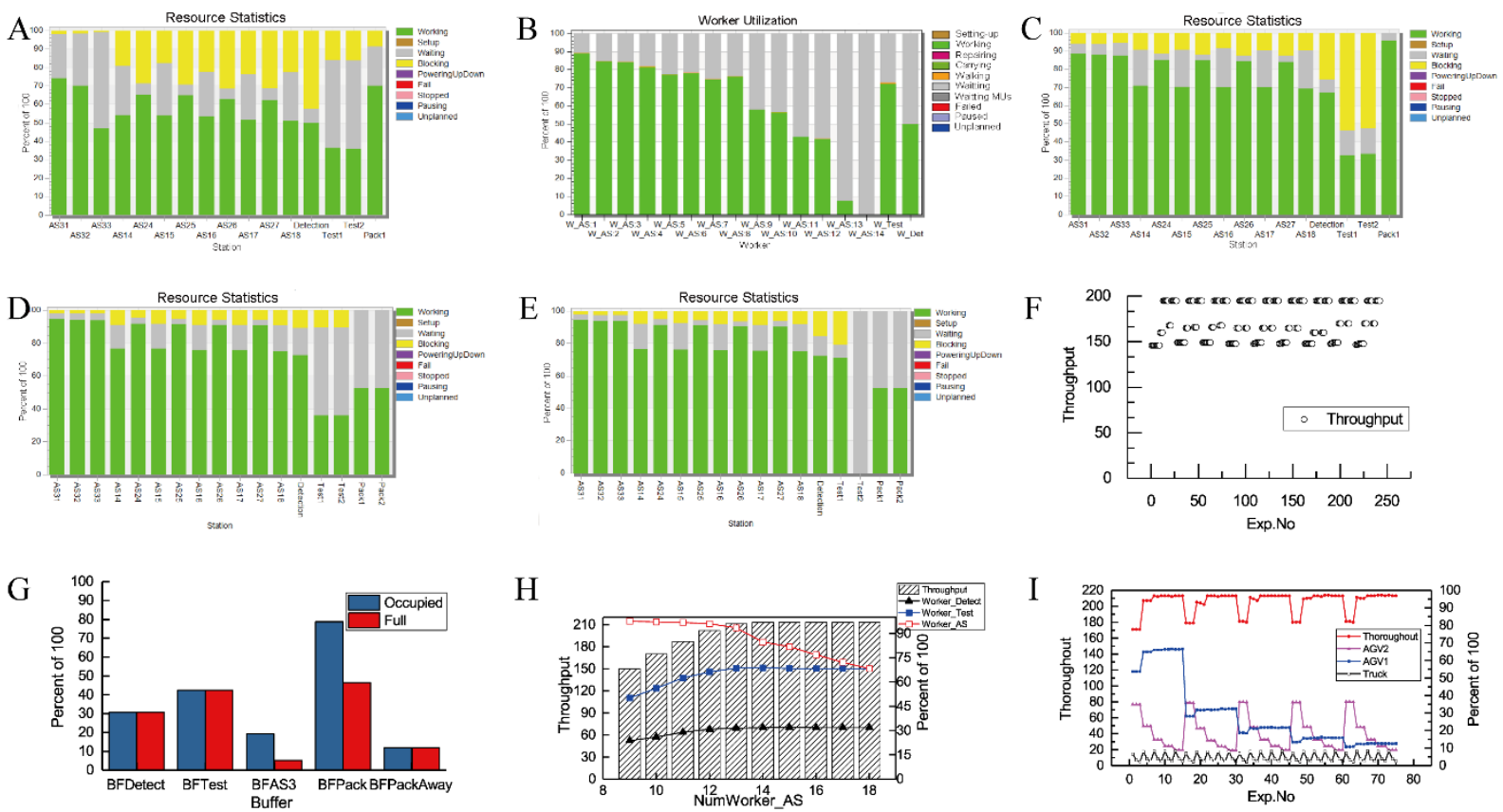

Figure 4: Optimization of production configuration: A) Facility utilization in the preliminary simulation, B) Worker utilization in the preliminary simulation, C) Facility utilization after the buffer optimization, D) Facility utilization after the bottleneck optimization, E) Facility utilization after the workstation adjustment, F) The throughput of the first stage experiment, G) Utilization of each buffer at the optimal capacity, H) Throughput and labour intensity for the different worker configurations, I) Throughput and vehicle utilization for the different vehicle configurations.

The experimental data shows that for the number of trucks, when the vehicle configurations increased from $5,5,1$ to $5,5,3$, and from $1,3,1$ to $1,3,3$, the throughput did not increase. This indicates that one truck can meet the production requirements. In addition, when one truck was used, the truck utilization was $8.2 \%$, which was relatively low. Therefore, the optimal number of trucks was determined to be one.

For AGV1, its quantity increased from 1 to 5, and the maximum output and utilization data are shown in Table IV. As shown in the table, one AGV vehicle could meet the production demand. However, considering the running time and failure occurrences, the optimal number of AGV1 vehicles was identified as two.

The experimental results for AGV2 vehicles are shown in Table V. Similar to the analysis of AGV1 vehicles, the number of AGV2 vehicles was identified as three.

\section{RESULTS}

The workshop layout with the smallest logistics volume was obtained by using a GA. In addition, the workshop performance was improved through simulation modelling and 
optimization of the entire production process. The comparison of the production configuration before and after optimization is shown in Table VI.

Table IV: The vehicle usage for AGV1.

\begin{tabular}{|c|c|c|c|}
\hline Quantity & $\begin{array}{c}\text { Running } \\
\text { time (h) }\end{array}$ & $\begin{array}{c}\text { Utilization } \\
(\%)\end{array}$ & $\begin{array}{c}\text { MaxOut } \\
\text { put }\end{array}$ \\
\hline 1 & 4.66 & 66.61 & 213 \\
\hline 2 & 2.27 & 21.73 & 213 \\
\hline 3 & 1.52 & 21.79 & 213 \\
\hline 4 & 1.12 & 15.92 & 213 \\
\hline 5 & 0.88 & 12.56 & 213 \\
\hline
\end{tabular}

Table V: The vehicle usage for AGV2.

\begin{tabular}{|c|c|c|c|}
\hline Quantity & $\begin{array}{c}\text { Running } \\
\text { time (h) }\end{array}$ & $\begin{array}{c}\text { Utilization } \\
(\%)\end{array}$ & $\begin{array}{c}\text { MaxOut } \\
\text { put }\end{array}$ \\
\hline 1 & 5.58 & 79.71 & 180 \\
\hline 2 & 3.37 & 48.22 & 210 \\
\hline 3 & 2.28 & 32.61 & 213 \\
\hline 4 & 1.72 & 24.57 & 213 \\
\hline 5 & 1.37 & 19.62 & 213 \\
\hline
\end{tabular}

As seen from Table VI, after optimization, the allocation of the main production resources in the workshop was adjusted accordingly. The optimal buffer allocation for the major workstations was determined. A packaging workstation was added, and a testing workstation was reduced. The number of logistics vehicles was adjusted and verified. Finally, the monthly output increased from 150 to 213 , which is $42.0 \%$ higher than that before optimization.

In addition, the workstation utilization increased greatly, and the blockages decreased significantly, as shown in Fig. 5 A. Moreover, the worker utilization was improved, and the labour intensity was balanced, as shown in Fig. 5 B.

Table VI: Production resource allocation and throughput comparison before and after optimization.

\begin{tabular}{|c|c|c|c|c|c|c|c|c|c|c|c|}
\cline { 2 - 13 } \multicolumn{1}{c|}{} & \multicolumn{4}{c|}{ Buffer } & \multicolumn{2}{c|}{ Station } & Worker & \multicolumn{3}{c|}{ Vehicle } \\
\cline { 2 - 12 } \multicolumn{1}{c|}{} & Detect & Test & AS3 & Pack & PackAway & Pack & Test & W_AS & AGV1 & AGV2 & Truck \\
\hline Before & 0 & 0 & 0 & 0 & 0 & 1 & 2 & 14 & 2 & 2 & 2 \\
\hline After & 1 & 1 & 2 & 2 & 1 & 2 & 1 & 14 & 2 & 3 & 1 \\
\hline
\end{tabular}
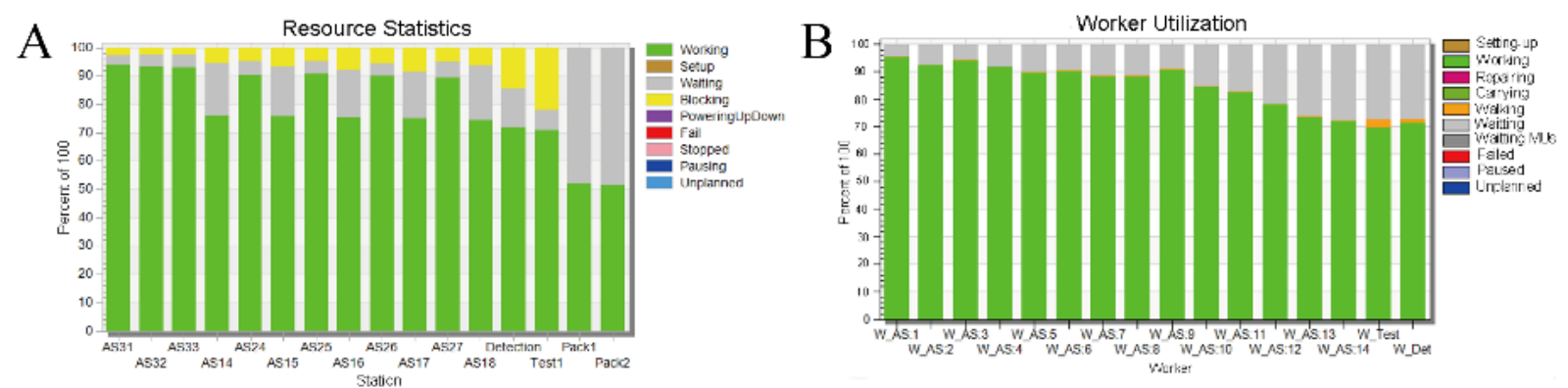

Figure 5: Workshop performance after optimization: A) Facility utilization after optimization, B) Worker utilization after optimization.

\section{CONCLUSIONS}

This research optimized the workshop layout, proposed a general modelling method for workshop logistics processes, and optimized an entire production process and a major production configuration. The purpose of this paper is to provide a comprehensive, efficient and accurate simulation optimization methodology and decision guidance for workshop layout, production performance and production resource allocation under a flexible production environment through DES.

This study shows that an accurate simulation model that describes complex production processes can be established so that the entire production process can be optimized and that more production resource allocation can be determined through DES. The second main finding is that a logistics simulation model of a complex workshop can be developed 
efficiently through the proposed general modelling method. These findings indicate that DES can provide comprehensive, efficient and accurate guidance for workshop planning and design.

However, this study was limited to a situation where the transport vehicles could only perform one task at the same time. If multiple tasks need to be performed, the dynamic task allocation strategies must be further developed.

The proposed general modelling method of the workshop logistics processes could be used in the logistics simulation design of other workshops, and the case study of a comprehensive assembly shop simulation and optimization can provide a reference for the planning of other workshops.

\section{ACKNOWLEDGEMENT}

The authors acknowledge funding from the Science and Technology Planning Projects of Shenyang (Project No. Z17-7-002).

\section{REFERENCES}

[1] Zhou, J.; Li, P.; Zhou, Y.; Wang, B.; Zang, J.; Meng, L. (2018). Toward new-generation intelligent manufacturing, Engineering, Vol. 4, No. 1, 11-20, doi:10.1016/j.eng.2018.01.002

[2] Yang, H.-L.; Chang, T.-W.; Choi, Y. (2018). Exploring the research trend of smart factory with topic modeling, Sustainability, Vol. 10, No. 8, Paper 2779, 15 pages, doi:10.3390/su10082779

[3] Zhang, Z.; Wang, X.; Wang, X.; Cui, F.; Cheng, H. (2019). A simulation-based approach for plant layout design and production planning, Journal of Ambient Intelligence and Humanized Computing, Vol. 10, No. 3, 1217-1230, doi:10.1007/s12652-018-0687-5

[4] Mallikarjuna, K.; Veeranna, V.; Reddy, K. H. (2016). A new meta-heuristics for optimum design of loop layout in flexible manufacturing system with integrated scheduling, International Journal of Advanced Manufacturing Technology, Vol. 84, No. 9-12, 1841-1860, doi:10.1007/s00170-015$\underline{7715-9}$

[5] Amiri, M.; Mohtashami, A. (2012). Buffer allocation in unreliable production lines based on design of experiments, simulation, and genetic algorithm, International Journal of Advanced Manufacturing Technology, Vol. 62, No. 1-4, 371-383, doi:10.1007/s00170-011-3802-8

[6] Samanta, S.; Philip, D.; Chakraborty, S. (2018). Bi-objective dependent location quadratic assignment problem: Formulation and solution using a modified artificial bee colony algorithm, Computers \& Industrial Engineering, Vol. 121, 8-26, doi:10.1016/j.cie.2018.05.018

[7] Matai, R. (2015). Solving multi objective facility layout problem by modified simulated annealing, Applied Mathematics and Computation, Vol. 261, 302-311, doi:10.1016/ j.amc.2015.03.107

[8] Yang, T.; Kuo, Y.; Su, C.-T.; Hou, C.-L. (2015). Lean production system design for fishing net manufacturing using lean principles and simulation optimization, Journal of Manufacturing Systems, Vol. 34, 66-73, doi:10.1016/j.jmsy.2014.11.010

[9] Tang, M.; Qi, Y.; Zhang, M. (2017). Impact of product modularity on mass customization capability: An exploratory study of contextual factors, International Journal of Information Technology \& Decision Making, Vol. 16, No. 4, 939-959, doi:10.1142/s0219622017410012

[10] Gong, D.; Tang, M.; Liu, S.; Li, Q. (2017). Reconsidering production coordination: A principalagent theory-based analysis, Advances in Production Engineering \& Management, Vol. 12, No. 1, 51-61, doi:10.14743/apem2017.1.239

[11] Subulan, K.; Cakmakci, M. (2012). A feasibility study using simulation-based optimization and Taguchi experimental design method for material handling-transfer system in the automobile industry, International Journal of Advanced Manufacturing Technology, Vol. 59, No. 5-8, 433443, doi:10.1007/s00170-011-3514-0

[12] Lindskog, E.; Vallhagen, J.; Berglund, J.; Johansson, B. (2016). Improving lean design of production systems by visualization support, Procedia CIRP, Vol. 41, 602-607, doi:10.106/j.procir.2016.01.004 
[13] Silva, M. (2018). On the history of discrete event systems, Annual Reviews in Control, Vol. 45, 213-222, doi:10.1016/j.arcontrol.2018.03.004

[14] Negahban, A.; Smith, J. S. (2014). Simulation for manufacturing system design and operation: Literature review and analysis, Journal of Manufacturing Systems, Vol. 33, No. 2, 241-261, doi:10.1016/j.jmsy.2013.12.007

[15] Rifai, A. P.; Dawal, S. Z. M.; Zuhdi, A.; Aoyama, H.; Case, K. (2016). Reentrant FMS scheduling in loop layout with consideration of multi loading-unloading stations and shortcuts, International Journal of Advanced Manufacturing Technology, Vol. 82, No. 9-12, 1527-1545, doi: $10.1007 / \mathrm{s} 00170-015-7395-5$

[16] Di Gironimo, G.; Lanzotti, A.; Peluso, F.; Renno, F. (2015). Virtual production planning of a high-speed train using a discrete event simulation based approach, International Journal on Interactive Design and Manufacturing, Vol. 9, No. 1, 65-75, doi:10.1007/s12008-013-0193-0

[17] Wang, Y.; Yang, O. (2017). Research on industrial assembly line balancing optimization based on genetic algorithm and Witness simulation, International Journal of Simulation Modelling, Vol. 16, No. 2, 334-342, doi:10.2507/ijsimm16(2)co8

[18] Leitão, P.; Mendes, J. M.; Bepperling, A.; Cachapa, D.; Colombo, A. W.; Restivo, F. (2012). Integration of virtual and real environments for engineering service-oriented manufacturing systems, Journal of Intelligent Manufacturing, Vol. 23, No. 6, 2551-2563, doi:10.1007/s10845$\underline{011-0591-8}$

[19] Qin, W.; Zhang, J.; Sun, Y. (2013). Multiple-objective scheduling for interbay AMHS by using genetic-programming-based composite dispatching rules generator, Computers in Industry, Vol. 64, No. 6, 694-707, doi:10.1016/j.compind.2013.03.009

[20] Yang, B.; Chen, W.; Lin, C. (2017). The algorithm and simulation of multi-objective sequence and balancing problem for mixed mode assembly line, International Journal of Simulation Modelling, Vol. 16, No. 2, 357-367, doi:10.2507/ijsimm16(2)co10

[21] Grabowik, C.; Kalinowski, K.; Cwikla, G.; Niemiec, K.; Paprocka, I. (2018). A computer simulation as a tool for a production system analysis and optimization, IOP Conference Series: Materials Science and Engineering, Vol. 400, No. 2, Paper 022033, 16 pages, doi:10.1088/1757$\underline{899 X / 400 / 2 / 022033}$

[22] Supsomboon, S.; Vajasuvimon, A. (2016). Simulation model for job shop production process improvement in machine parts manufacturing, International Journal of Simulation Modelling, Vol. 15, No. 4, 611-622, doi:10.2507/ijsimm15(4)3.352

[23] Andrade-Gutierrez, E. S.; Carranza-Bernal, S. Y.; Hernandez-Sandoval, J.; Gonzalez-Villarreal, A. J.; Berber-Solano, T. P. (2018). Optimization in a flexible die-casting engine-head plant via discrete event simulation, The International Journal of Advanced Manufacturing Technology, Vol. 95, No. 9-12, 4459-4468, doi:10.1007/s00170-017-1562-9

[24] Huang, D. M.; Zhang, G. J.; Shi, S. X. (2012). Research on simulation and optimization of facility layout in flexible manufacturing workshop, Applied Mechanics and Materials, Vol. 108, 24-29, doi:10.4028/www.scientific.net/AMM.108.24

[25] Hodoň, R.; Kovalský, M.; Gregor, M.; Grznár, P. (2018). New approaches in production scheduling using dynamic simulation, IOP Conference Series: Materials Science and Engineering, Vol. 393, No. 1, Paper 012023, 11 pages, doi:10.1088/1757-899X/393/1/012023

[26] Supsomboon, S.; Varodhomwathana, T. (2017). Robot and plant simulation for automotive part production process design: A case study, International Journal of Simulation Modelling, Vol. 16, No. 4, 617-629, doi:10.2507/ijsimm16(4)5.397

[27] Pillai, V. M.; Hunagund, I. B.; Krishnan, K. K. (2011). Design of robust layout for dynamic plant layout problems, Computers \& Industrial Engineering, Vol. 61, No. 3, 813-823, doi:10.1016/j.cie.2011.05.014

[28] Zhang, H.; Beltran-Royo, C.; Wang, B.; Ma, L.; Zhang, Z. (2016). Solution to the quadratic assignment problem using semi-lagrangian relaxation, Journal of Systems Engineering and Electronics, Vol. 27, No. 5, 1063-1072, doi:10.21629/jsee.2016.05.14 